\title{
A two tier architecture for Local Energy Market Simulation and Control *
}

\author{
Andrade, Rui ${ }^{1[0000-0003-2356-3706]}$, Garcia-Rodriguez, \\ Sandra $^{2[0000-0002-5352-2510]}$, Praca, Isabel ${ }^{1[0000-0002-2519-9859]}$, and Vale, \\ Zita $^{1[0000-0002-4560-9544]}$ \\ 1 ISEP/GECAD ***, Porto, Portugal \\ \{rfaar,icp,zav\}@isep.ipp.pt \\ 2 CEA, LIST, Data Analysis and Systems Intelligence Laboratory, 91191, Gif Sur \\ Yvette, France sandra.garciarodriguez@cea.fr
}

\begin{abstract}
This paper addresses energy management and security having as basis sensing and monitoring of cyber-physical infrastructure of consumers and prosumers, and their participation in the Local Energy Market (LEM). The vision is to create a layered multi-agent framework that brings a complete view of the cyber-physical system of LEM participants, and provides optimization and control of energy for said participants. The proposed system is separated into a Market layer and a Cyber-Physical layer, each of them providing different services. The cyber-physical layer, represented by SMARTERCtrol system, provides Data Monitoring and Optimized Energy Control of individual building resources. The Market layer, represented by LEM Multi-Agent System, provides Negotiation, Forecasting, and Trust Evaluation. Both systems work together to provide and integrate a tool for simulation and control of LEM.
\end{abstract}

Keywords: Multi-Agent Systems · Energy · Optimization · Forecasting

\section{Introduction}

According to European Commission [4] the share of renewables in Power and Energy Systems (PES) could reach $50 \%$ by 2030 with an important contribution from variable sources. This sets significant challenges to distribution grids since large part of the renewables will be implemented at household level. Furthermore, the European parliament proposal for the regulation of the internal Energy Market (EM) suggests that the role of consumers in future PES will be central [7]. The developed solutions and technology should encourage and enable consumers to take part in the energy transition and participate in EM transactions. One of the main reasons for the need of an active participation from consumers and prosumers is the current inaccuracies in the balance settlement,

* This work has received funding from National Funds through FCT (Fundaçao da Ciencia e Tecnologia) under the project SPET - 29165, call SAICT 2017. 
as it happens in most European countries, since much of the load is profiled because the smart meters roll out is not completed yet [20]. While smart meters facilitate accurate and efficient balance settlement, the development of metering and control opportunities needs to be addressed carefully in regulatory rules. For example, the handling of imbalances caused by control actions made by non-balance responsible parties is one of these issues [24].

Information exchange structures and models to enable interaction between local, retail and wholesale EM are urgently needed, including effective proposals on how local markets should be taken into account by regulatory aspects. In this context, the use of simulation tools and the study of the different market mechanisms and the relationships among their stakeholders, becomes essential.

A local market can be seen as a place where individual consumers and prosumers meet to trade energy in a neighborhood environment [12]. A major goal for local EM is to contribute in a decentralized PES [25]. Distributed Energy Resources (DER) have increased the complexity of PES radically and therefore flexibility is emerging as the most crucial element in the system. Advantage of local markets is not only that self-generation can be consumed locally but it also strengthens local distribution networks and provides new opportunities for local industry and regional businesses.

The practical implementation and widespread of local EM is, however, highly dependent on the available physical structure. One of the main drivers in this scope is the development already achieved in Smart Grid (SG) technology. Sensor networks are one of the most suitable technologies for SG due to their low-cost, collaborative and longstanding nature. Wireless sensor Networks (WSNs) can enable both utilities and customers to transfer, monitor, predict, measure, and manage energy usage effectively. Thus, WSN can revolutionize the current electric power infrastructure by integrating information and communication technologies (ICT) $[27,11]$. Such a heavy dependence on ICT networking inevitably surrenders SG to potential vulnerabilities. Thus, security emerges as a critical issue because millions of heterogeneous devices (e.g., sensors, meters) are interconnected via communication networks.

Multi-Agent Systems (MAS) [28] are particularly well suited for the analysis of complex interactions in dynamic systems, such as energy market [10]. Some of the key advantages of MAS are the facilitated inclusion of new models, market mechanisms, and types of participants, as well as the ability to resolve problems in a distributed way [28]. Several modeling tools for simulating EM have emerged, such as AMES [16], EMCAS [14], ABEMS [8] and MASCEM [22].

In this paper we present a two layer approach to model the LEM. These layers are the MAS energy management system (SMARTERCtrol) that performs data collecting and optimized control of the grid resources, and the Local Energy Market Multi-Agent System (LEMMAS) that performs energy negotiating among local participants, provides energy forecasting and trust evaluation for the negotiations.

The remain of the paper is organized as follows. Section 2 describes the Local Energy Market; and presents the LEMMAS simulation model and its integra- 
tion with the SMARTERCtrol systems. Section 3 details the LEMMAS services and their importance fore the system. Section 4 details the SMARTERCtrol for control and optimization. Section 5 presents the conclusions of this work.

\section{Local Energy Market}

The energy landscape is changing at a rapid rate, renewable energy sources created the opportunity for traditional consumers to become producers of part, or in some cases all, of their energy needs. However this increase in what is called Distributed Energy Resources (DER) can create large and unpredictable fluctuation of energy loads in the electric grid.

The Local Energy Market (LEM) [17] is an emergent market model that is aimed at solving the problems inherent to the Renewable and DER, such as unpredictability of energy generation. Participants in the LEM who are generating energy but not consuming it can chose to sell it in the LEM.

The key features of the LEM are the flexibility it provides in terms of creating distributed and efficient energy consumption, and the opportunity, created for traditional consumers, to participant in a kind of energy market. In this section, we present an overview of the LEMMAS simulation model and detail how it can benefit from the integration with the multi-agent optimization and control system (SMARTERCtrol).

In a previous work [19], the authors present a MAS model to simulate and study the LEM. This model is composed of three kinds of agents:

- Sensor agent: Representing the cyber-physical system;

- Participant agent: Representing participants in the negotiation;

- Market Interactions Manager (MIM): Representing the negotiation manager.

Participants in the LEM can be either consumers, producers or prosumers (consumers with some form of generation, e.g. an household with solar panels). Each participant is modeled accordingly to its role with the corresponding sensor agents representing its cyber-physical system.

In the LEMMAS simulation model the agents follow an hierarchical structure in their communication. The sensor agents only communicate with their respective participant agent, sending updated values for consumption, generation, battery charge, etc. The participant agents sends a proposal to the MIM. And the MIM acts as central authority in the negotiation: sends messages to all participants in other to enable the negotiation process. Each participant agent would have the necessary number of sensor agents to model its cyber-physical system.

Figure 2 illustrates the architecture proposed in this article. Two layers are evidenced in this architecture, the Local Market Layer and the Cyber-Physical Layer. These layers should have independent responsibilities but be complementary to each other. On the Cyber-Physical Layer each household or building is represented and supported by the SMARTERCtrol system. The SMARTERCtrol system is responsible for providing the services of Data Monitoring, in 


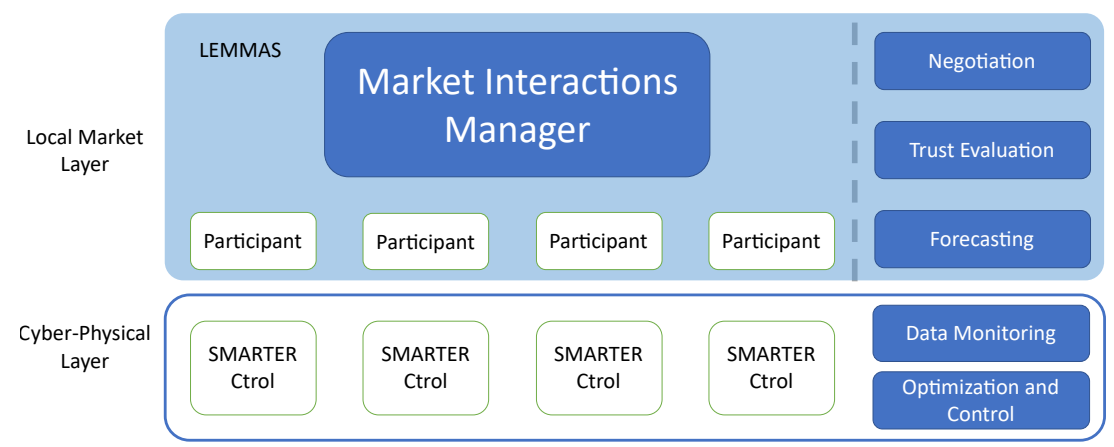

Fig. 1. Local Market two Layer Architecture

terms of consumption, generation, and other data sources like energy storage (if available); and the optimized control of energy resources. The Market Layer is represented by the LEMMAS system, composed of the group of Participants and the Market Interactions Manager. The LEMMAS provides the services of Negotiation, Trust Evaluation, and Forecasting.

The household or building is represented in both layers but in different ways, in the Local Market layer this representation is made by the participant agent that is able to submit proposals to buy or sell energy in the LEM. The proposals, as well as the forecasting service, are integrated with the SMARTERCtrol of each participant in order to make use of the current and historical data collected by the SMARTERCtrol. Lastly, the integration with the SMARTERCtrol system can also happen as a result of the negotiation. That is, after an energy transaction is agreed upon in the Local Market, this information must be sent to the SMARTERCtrol system of the participants involved in said transaction. With the obtained information the SMARTERCtrol system must perform the necessary optimization and control operations in order to allow the desired energy transaction.

The SMARTERCtrol system brings improvements to the LEM's performance, reliability and resilience, by performing two different kinds of control:

1. Scheduling control based on forecasted data: these forecasts are related to the microgrid devices (such as consumption schedules of home appliances, battery levels, etc.) or other forecasted information for generation (e.g. solar radiation, wind speed, etc.) to plan the use of the generated flexibility, based on the result of the MAS optimization. This type of control may handle uncertainties in forecasts. It can also adapt itself to possible changes in grid topology by applying receding horizon control along the time in which the planning activity is undertaken; for instance, in case control relies on forecasted information, initial planning can be done with great advance (e.g. the day ahead) and then refined when getting closer to real-time.

2. Real-time control: It is based on real-time information provided by sensors (e.g. power quality analysers, voltage sensors, etc.) to monitor the status of 
all nodes of the grid on a real-time basis. The scope is to make sure grid parameters are always within statutory limits and cope with critical conditions. These two types of control are both required for an effective management of the smart-building, but the type of information that they need to fulfil their duties differs. While the planning control requires forecasted information and data about scheduled or expected use of smart grid technologies, real-time control only needs actual readings of grid monitoring devices.

With the addiction of the SMARTERCtrol system, the sensor agents are no longer used. The SMARTERCtrol acts as an aggregator of all sensor data that can pass this information to the corresponding participant agent, this is evidenced in Figure 2.

In turn the participant agent sends information detailing the result of the negotiations in the LeM to the SMARTERCtrol. Thus allowing the SMARTERCtrol to make the necessary control adjustments and optimizations to enable the desired outcome. For example, a participant agent might agree to sell energy to another LEM participant. In this scenario the SMARTERCtrol system should adjust the necessary control flow to make that transaction happen.

\section{LEMMAS Services}

The Local Energy Market Multi-Agent System (LEMMAS) takes the responsibility of providing the platform necessary for the negotiation between the participants. However the negotiation needs to be supported with other functionalities. For this reason the LEMMAS provides two complementary services to the negotiation, then being forecasting, and trust evaluation.

\subsection{Negotiation}

The main service provided by the LEMMAS is the service of negotiation. The negotiation in LEMMAS is made in Mediated Contracts, that is, there is a thirdparty that plays the role of a mediator and manages the negotiation. These Mediated Contracts follow a Double Sided Auction model that defines the Market Clearing price, and is performed regular intervals in order to keep up with the market needs.

In the LEMMAS this mediator is the Market Interactions Manager (MIM) agent. When performing the negotiation, this agent also takes advantage of the Trust Evaluation service in order to only allow trusted participants in the negotiation.

\subsection{Forecasting}

The forecasting service provided by the LEMMAS is used internally by the Trust Evaluation Service and by the SMARTERCtrol system as well. The forecasting module includes several methods and a set of strategies for day-ahead and 
hour-ahead forecasting. Indeed, one of the most important targets of the energy operators is to be able to have a better control on the energy consumption and also being prepared for the amount of energy demand in the following hours or days. In order to provide consumption and generation forecasting for different short and long terms, this service should make use of various forecasting methods: Artificial Neural Networks [5], Support Vector Machines [26], Fuzzy Rule Based Systems [13], Reinforcement Learning [2], Time Series Analysis [6] and Ensemble Methods [23]. Ensemble Methods, in particular, have already shown a good performance in a precious work [23].

\subsection{Trust Evaluation}

In any kind of negotiation trust is a crucial factor. Negotiating with an non trusted party may jeopardize the negotiation. The Trust Evaluation service provides an important security layer for the participants. The idea for the trust mechanism, dynamic profiles of each participant are defined, based on previous market negotiations and using the forecasting service. These profiles are then used according to the context, current weather conditions, time, date, etc., to support the analysis of the feasibility of submitted proposals. LEMMAS follows what is called an Institutional Trust model [18], that means there exists a centralized entity responsible for applying the trust model. In the case of LEMMAS this role is played by the MIM agent that acts as the centralized institution, and enforces the Trust model [1].

\section{Multi-Agent Optimization and Control}

This section presents a multi-agent optimization and control system (SMARTERCtrol) for microgrids. Taking into account the constraints, limitations and user preferences, its goal is to perform an optimized management of the self-controlled resources of a microgrid. Furthermore, this system is connected to the forecasting service described previous section 3.2. This way SMARTERCtrol enriches the quality of decisions by also considering some consumption/generation forecasts in the decision making.

SMARTERCtrol is composed by two main modules, the optimization module and the control one. Each of them counts with a different multi-agent system model and deploys their own agents which are continuously communicating to coordinate:

- Control module: agents are in charge of controlling the existing devices. This module deploys one agent(Control Device) per device of the microgrid plus a central coordinator one (Control Node). Their main tasks are collecting the forecasts or actual observations, communicate with the optimization module and get the optimized schedules, and convert the optimized schedules received from the optimization module into orders that are then sent to the self controlled devices. It makes sure that the devices follow such schedules. 
Note that in case of real time control, schedules would have just one instant period of time (no planning needed).

- Optimization: optimization agents collaborate to implement a distributed optimization algorithm based on message passing [15]. Relying on the information (forecasts, observations, etc.) provided by the control module, this module computes the best schedules and sends them to it. Two kind of agents are used in this module, Optimization Node and Optimization Device. The number of agents deployed will depend on the microgrid scenario.

Fig. 4 shows a schema of both modules as well as the main interactions among their agents. The concrete algorithms that each module follows are described below.

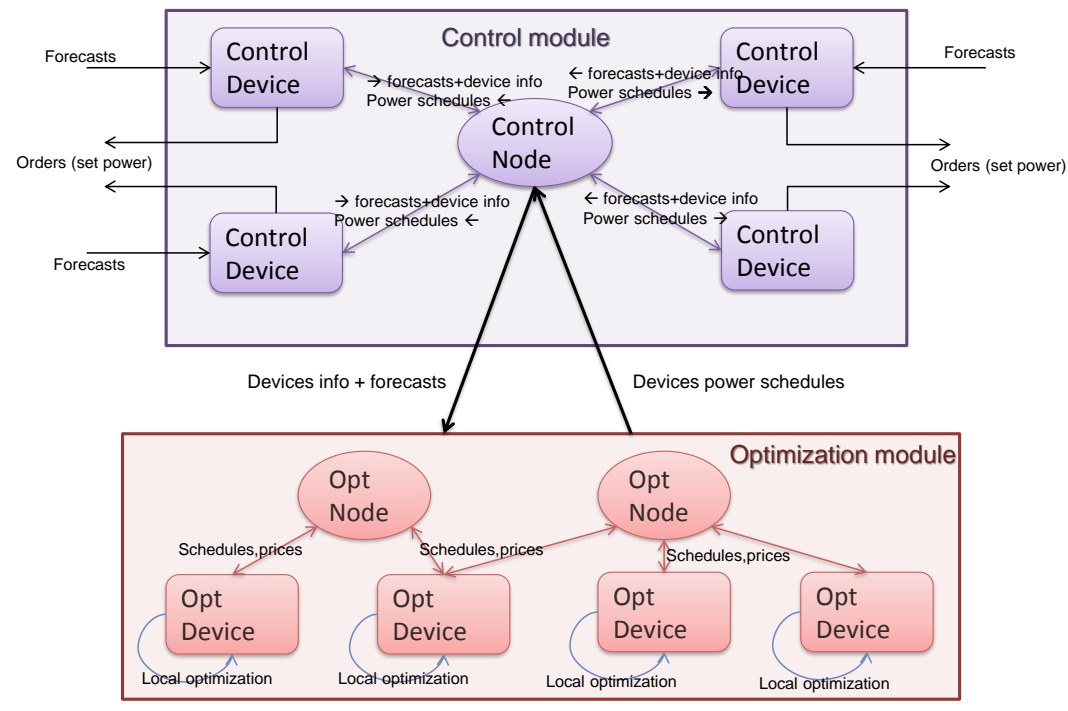

Fig. 2. SMARTERCtrol optimization and control modules, interactions

\subsection{Control Module Algorithm}

This module connects the platform with the external microgrid. As Algorithm 1 shows, this module runs a continuous loop in which every $T$ time the same steps from 2 to 12 are repeated. $T$ is set a priori according to user preferences and microgrid definition. Algorithm 1 also shows the interactions between agents and modules, where " $A \rightarrow B$ " means that $A$ sends the message $m s g$ to $B$. 


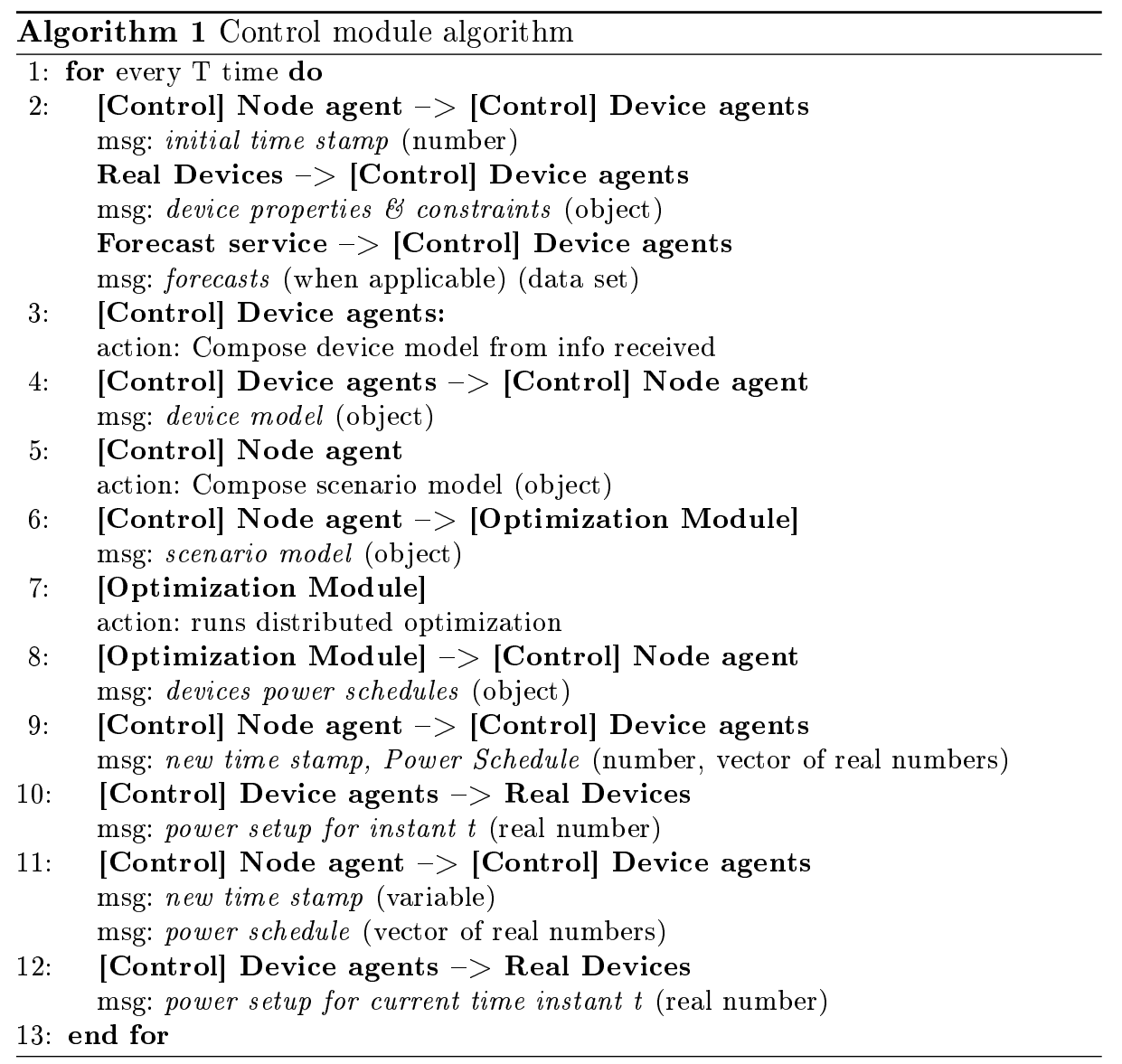

\subsection{Optimization Module}

Implemented with an agent oriented architecture already described in [9], this module performs the optimization task. It is based on the message passing algorithm adapted to energy management, and proposed by [15]. Such approach relies on the alternating direction method of multipliers (ADMM), which is an algorithm that solves convex optimization problems by breaking them into smaller pieces so that they will be then easier to handle [3]. [15]'s model considers two types of elements in a given grid, namely: the devices and the nets (called $o p$ timization devices and optimization nodes respectively in our approach). The devices $D$ (i.e. generators, fixed loads, deferrable loads, alternate direct current transmission lines, storages, etc.) have their own constraints and objectives. Devices are connected to each other by means of a net (i.e. bus), which also has its own objectives. In the same way, nets are connected through double terminal devices, such as transmission lines. The model tries to minimize the global 
network objective subject to the device and net constraints, over a given time horizon.

[15] use a proximal message passing algorithm to resolve this optimization problem. It is an iterative algorithm where at each step, each device: (i) exchanges simple messages with its neighbours in the network; and then (ii) it solves its own optimization problem by minimising a local objective function, augmented by the messages it has received. The authors showed that their approach converges to a solution when the devices objective and constraints are convex. The method is completely decentralized and needs no global coordination other than synchronizing iterations; the problems to be solved by each device can be typically solved efficiently and in parallel according to the authors.

In this work, we considered evolutionary optimization algorithms [29] to solve such local optimization. The using of this kind of algorithms is recurrent in literature since they are proved to be good approaches to solve microgrid problems [21]. For instance, their flexibility allows to handle the most hard constraints in the optimization process. Moreover, their operators can be easily modified to improve the algorithm performance in the specific problem to solve.

\subsection{Systems Connection}

Lastly the two systems, LEMMAS and SMARTERCtrol, are connected at the Forecasting level. The Control nodes in SMARTERCtrol make use of the Forecasting values obtained from LEMMAS' Forecasting Module, as shown in Figure 4.3. This architecture brings advantages at the level of separation of concerns into: Simulation, Control and Optimization. The two domains can be developed separately, but have the advantage of working together, taking advantages of each others strengths. The SMARTERCtrol taking Control of real devices gains access to a Simulation environment that uses the Real-time monitoring data with strategies optimized for the context, and provides SMARTERCtrol with the forecasts necessary.

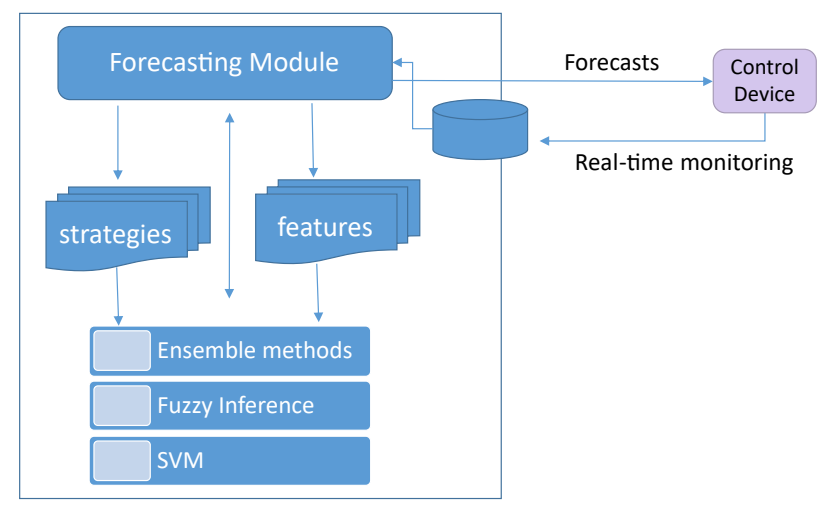

Fig. 3. Forecasting-Module with SMARTERCtrol 


\section{Conclusion}

As it was discussed in this article energy markets are going through a shift of paradigm, from centralized production to distributed production of energy. This changes bring benefits in terms of flexibility, for consumers who can afford sell generation, as well as possible benefits to the environment with the increased use of renewable energy sources. However this benefits come with some negative aspects: the increase of distributed production makes the energy grid more complex and hard to manage. The ever increasing usage of renewable energy sources, e.g. solar, creates unpredictable energy fluctuation, and the moments of higher production do not always correspond with the moments of higher consumption needs. Fore these reasons, having systems capable of simulating and controlling the energy grids will always be needed.

In this article we proposed a two tier architecture to simulate and control the Local Energy Market (LEM). The two layers in this architecture are the Local Market Layer and the Cyber-Physical Layer. Such separation of concerns brings benefits in terns of development by dividing systems in smaller more specialized components, but the system remain tightly integrated as a full stack solution for LEM simulation and control. This was possible due to the usage of services in each layer that can integrate with services from the other layer whenever necessary.

The Cyber-Physical Layer corresponds to the SMARTERCtrol system, representing a single household or building. The SMARTERCtrol system provides the services of Data Monitoring in terms of consumption, generation, and other data sources (if available); and for the optimized energy control.

The Market Layer corresponds to the LEMMAS system. It represents the LEM as a whole with several participants, each one connected with its own SMARTERCtrol system and Market Interactions Manager agent, acting as the central authority of these market. The LEMMAS system provides the services of Negotiation, Trust Evaluation, and Forecasting.

The integration between the two layers is as follows. The Negotiation service gives each participant the opportunity to make proposals to buy or sell energy in the market. Such proposals are made taking into consideration the participant needs reported by the Data Monitoring service. The Negotiation service uses the Trust Evaluation service to verify which participants should be allowed in to participant in the market. The Trust Evaluation takes into consideration the values forecasted by the Forecasting service considering data from the Data Monitoring service.

Lastly, the Negotiation service communicates its results to the Optimization and control service so that this service can make the necessary changes in the grind to make the energy transactions agreed in the Local Market.

With this new approach the LEMMAS sensor agents are replaced by the SMARTERCtrol. The benefits of this new approach are twofold. First, the SMARTERCtrol system acts a central point that aggregates the data from all sensor readings and makes it available for other services. And second, the SMARTERCtrol system can perform the necessary control and optimization 
operations to realize the energy transactions agreed the local energy Market negotiation process.

\section{References}

1. Andrade, R., Pinto, T., Praça, I.: A Multi-Agent System Simulation Model for Trusted Local Energy Markets (2019)

2. Andrade, R., Pinto, T., Praça, I., Vale, Z.: UCB1 Based Reinforcement Learning Model for Adaptive Energy Management in Buildings. In: Rodríguez, S., Prieto, J., Faria, P., Kłos, S., Fernández, A., Mazuelas, S., Jiménez-López, M.D., Moreno, M.N., Navarro, E.M. (eds.) Distributed Computing and Artificial Intelligence, Special Sessions, 15th International Conference. pp. 3-11. Springer International Publishing, Cham (2019)

3. Boyd, S., Parikh, N., Chu, E., Peleato, B., Eckstein, J.: Distributed Optimization and Statistical Learning via the Alternating Direction Method of Multipliers. Foundations and Trends in Machine Learning 3(1), 1-122 (2011)

4. Commission, E.: Horizon 2020 Work Programme 2018-2020. Secure, clean and efficient energy, vol. 2020 (2018), http://ec.europa.eu/research/participants/ data/ref/h2020/wp/2018-2020/main/h2020-wp1820-energy $\left\{\backslash \_\right\} e n . p d f$

5. Deb, C., Eang, L.S., Yang, J., Santamouris, M.: Forecasting diurnal cooling energy load for institutional buildings using artificial neural networks. Energy and Buildings 121, $284-297$ (2016)

6. Ediger, V.Ş., Akar, S.: Arima forecasting of primary energy demand by fuel in turkey. Energy policy 35(3), 1701-1708 (2007)

7. European Comission: Regulation on the internal market for electricity 0379(2016) (2016), https://ec.europa.eu/energy/sites/ener/files/documents/1\{\_ \}en $\left\{\backslash_{-}\right\}$act $\left\{\backslash_{-}\right\}$part $1\left\{\backslash_{-}\right\}$v9.pdf

8. Garcia-Rodriguez, S., Gomez-Sanz, J.J.: Robust decentralised agent based approach for microgrid energy management. In: Proceedings of the 18th International Conference on Autonomous Agents and MultiAgent Systems. pp. 772-780. International Foundation for Autonomous Agents and Multiagent Systems (2019)

9. Garcia-Rodriguez, S., Sleiman, H.A., et al.: A multi-agent system architecture for microgrid management. In: International Conference on Practical Applications of Agents and Multi-Agent Systems. pp. 55-67. Springer (2016)

10. Gomez-Sanz, J.J., Garcia-Rodriguez, S., Cuartero-Soler, N., Hernandez-Callejo, L.: Reviewing microgrids from a multi-agent systems perspective. Energies $7(5)$, $3355-3382$ (2014)

11. Grilo, A., Sarmento, H., Nunes, M., Gonçalves, J., Pereira, P., Casaca, A., Fortunato, C.: A wireless sensors suite for smart grid applications. In: 1st International Workshop on Information Technology for Energy Applications (2012)

12. Ilic, D., Da Silva, P.G., Karnouskos, S., Griesemer, M.: An energy market for trading electricity in smart grid neighbourhoods. In: 2012 6th IEEE international conference on digital ecosystems and technologies (DEST). pp. 1-6. IEEE (2012)

13. Jozi, A., Pinto, T., Praça, I., Silva, F., Teixeira, B., Vale, Z.: Energy consumption forecasting based on Hybrid Neural Fuzzy Inference System. In: 2016 IEEE Symposium Series on Computational Intelligence (SSCI). pp. 1-5 (2016). https://doi.org/10.1109/SSCI.2016.7849859

14. Koritarov, V.S.: Real-world market representation with agents. IEEE Power and Energy Magazine 2(4), 39-46 (2004) 
15. Kraning, M., Chu, E., Lavaei, J., Boyd, S.: Dynamic network energy management via proximal message passing. Foundations and Trends in Optimization 1(2), 73126 (2014)

16. Li, H., Tesfatsion, L.: Development of Open Source Software for Power Market Research: The AMES Test Bed. Iowa State University, Department of Economics, Staff General Research Papers 2 (2009)

17. Mengelkamp, E., Diesing, J., Weinhardt, C.: Tracing local energy markets: A literature review. it-Information Technology 61(2-3), 101-110 (2019)

18. Pinyol, I., Sabater-Mir, J.: Computational trust and reputation models for open multi-agent systems: a review. Artificial Intelligence Review 40(1), 1-25 (2013)

19. Praça, I., Ramos, S., Andrade, R., d. Silva, A.S., Sica, E.T.: Analysis and Simulation of Local Energy Markets. In: 2019 16th International Conference on the European Energy Market (EEM). pp. 1-5 (2019). https://doi.org/10.1109/EEM.2019.8916524

20. Ramos, S., Duarte, J.M., Duarte, F.J., Vale, Z.: A data-mining-based methodology to support mv electricity customers characterization. Energy and Buildings 91, 16 $-25(2015)$

21. Sanseverino, E.R., Di Silvestre, M.L., Ippolito, M.G., De Paola, A., Re, G.L.: An execution, monitoring and replanning approach for optimal energy management in microgrids. Energy 36(5), 3429-3436 (2011)

22. Santos, G., Pinto, T., Praça, I., Vale, Z.: MASCEM: Optimizing the performance of a multi-agent system. Energy 111, 513-524 (2016). https://doi.org/10.1016/j.energy.2016.05.127

23. Silva, J., Praça, I., Pinto, T., Vale, Z.: Energy consumption forecasting using ensemble learning algorithms. In: International Symposium on Distributed Computing and Artificial Intelligence. pp. 5-13. Springer (2019)

24. Smart Grid Task Force: Regulatory Recommendations for the Deployment of Flexibility - EG3 REPORT. Policy Report by Smart Grid Task Force, European Commission pp. 1-94 (January 2015), https://ec.europa.eu/energy/sites/ener/ files/documents/EG3Final- January2015.pdf

25. Van Der Schoor, T., Scholtens, B.: Power to the people: Local community initiatives and the transition to sustainable energy. Renewable and sustainable energy reviews 43, 666-675 (2015)

26. Vinagre, E., Pinto, T., Ramos, S., Vale, Z., Corchado, J.M.: Electrical energy consumption forecast using support vector machines. In: 2016 27th International Workshop on Database and Expert Systems Applications (DEXA). pp. 171-175. IEEE (2016)

27. Wang, W., Lu, Z.: Cyber security in the smart grid: Survey and challenges. Computer networks 57(5), 1344-1371 (2013)

28. Wooldridge, M.: An introduction to multiagent systems. John Wiley \& Sons (2009)

29. Zhang, J., Zhan, Z.h., Lin, Y., Chen, N., Gong, Y.j., Zhong, J.h., Chung, H.S.H., Li, Y., Shi, Y.h.: Evolutionary computation meets machine learning: A survey. IEEE Computational Intelligence Magazine 6(4), 68-75 (2011) 